Originalien

Z Rheumatol 2022 $81: 730-743$ https://doi.org/10.1007/s00393-021-01019-x Angenommen: 25. April 2021

Online publiziert: 11. August 2021

() Der/die Autor(en) 2021

\section{Redaktion}

U. Müller-Ladner, Bad Nauheim

U. Lange, Bad Nauheim

U. Kiltz $\cdot$ V. Buschhorn-Milberger ${ }^{1}$ K. Albrecht ${ }^{2} \cdot$ H.-J. Lakomek ${ }^{3} \cdot$ H.-M. Lorenz ${ }^{4} \cdot$ M. Rudwaleit ${ }^{5} \cdot$ M. Schneider ${ }^{6} \cdot$ H. Schulze-Koops ${ }^{7} \cdot$ X. Baraliakos $^{1} \cdot$ F. Behrens ${ }^{8}$. J. Brandt-Jürgens ${ }^{9} \cdot$ H. Haibel ${ }^{10} \cdot$ L. Hammel ${ }^{11} \cdot$ K. Karberg $^{12} \cdot$ H. Kellner ${ }^{13,14} \cdot$ D. Krause ${ }^{15} \cdot$ U. Lange $^{16} \cdot$ E. Märker-Herrmann ${ }^{17} \cdot$ D. Poddubnyy ${ }^{10} \cdot$ J. Sieper ${ }^{10}$. U. Syrbe ${ }^{10,18} \cdot$ J. Braun $^{1}$

'Rheumazentrum Ruhrgebiet, Herne, und Ruhr-Universität Bochum, Herne, Deutschland; ${ }^{2}$ Programmbereich Epidemiologie, Deutsches Rheuma-Forschungszentrum (DRFZ), Berlin, Deutschland; ${ }^{3}$ Universitätsklinik für Geriatrie, Johannes-Wesling-Klinikum Minden, Minden, Deutschland; ${ }^{4}$ Sektion für Rheumatologie, Medizinische Klinik V, Universitätsklinikum Heidelberg, Universität Heidelberg, Heidelberg, Deutschland; ${ }^{5}$ Klinik für Innere Medizin und Rheumatologie, Klinikum Bielefeld Rosenhöhe, Bielefeld, Deutschland; ' ${ }^{\circ}$ Poliklinik, Funktionsbereich und Hiller Forschungszentrum für Rheumatologie, Universitätsklinikum Düsseldorf, Heinrich-Heine-Universität Düsseldorf, Düsseldorf, Deutschland; ${ }^{7}$ Sektion Rheumatologie und Klinische Immunologie, Medizinische Klinik und Poliklinik IV, LMU-Klinikum München, Ludwig-Maximilians-Universität München, München, Deutschland; ${ }^{8}$ Abteilung Rheumatologie, Universitätsklinikum Frankfurt/Main, Goethe-Universität Frankfurt/Main, Frankfurt/Main, Deutschland; ${ }^{9}$ Rheumatologische Schwerpunktpraxis, Ärztehaus am Walter-Schreiber-Platz, Berlin, Deutschland; ${ }^{10}$ Medizinische Klinik für Gastroenterologie, Infektiologie und Rheumatologie, Charité Universitätsmedizin Berlin, Berlin University Alliance, Berlin, Deutschland; " DVMB - Deutsche Vereinigung Morbus Bechterew e. V., Schweinfurt, Deutschland; ${ }^{12}$ Rheumapraxis Steglitz, Berlin, Deutschland; ${ }^{13}$ Schwerpunktpraxis für Rheumatologie und Gastroenterologie, München, Deutschland; ${ }^{14}$ Abteilung Rheumatologie, Krankenhaus Neuwittelsbach, München, Deutschland; ${ }^{15}$ Rheumatologische Gemeinschaftspraxis, Gladbeck, Deutschland; ${ }^{16}$ Abteilung für Rheumatologie und Klinische Immunologie, Kerckhoff Klinik, Justus-Liebig-Universität Gießen, Bad Nauheim, Deutschland; ${ }^{17}$ Abteilung für Innere Medizin IV: Rheumatologie und Nephrologie, HELIOS Dr. Horst Schmidt Kliniken Wiesbaden, Wiesbaden, Deutschland; ${ }^{18}$ Rheuma Praxis Berlin, Berlin, Deutschland

\title{
Entwicklung von Qualitätsstandards für Patient*innen mit axialer Spondyloarthritis zum Einsatz in Deutschland
}

Die axiale Spondyloarthritis (axSpA) ist eine meist chronisch verlaufende entzündlich rheumatische Erkrankung des Achsenskeletts, die oft in den Sakroiliakalgelenken beginnt. Im Verlauf kommt es bei vielen Patient ${ }^{*}$ innen zu strukturellen knöchernen Veränderungen in der Wirbelsäule, initial in Form von Syndesmophyten. Auch periphere Gelenke und Enthesen können in unterschiedlichem Ausmaß betroffen sein, extramuskuloskeletale Manifestationen an Augen, Haut und Darm sind häufig $[1,2]$.

National und international gibt es zum Teil erhebliche Unterschiede in der Qualität der Gesundheitsversorgung von Patient*innen mit entzündlich rheumatischen Erkrankungen [3, 4].
Entsprechend wurden Defizite in der Versorgung von axSpA-Patient ${ }^{*}$ innen mit biologischen „disease-modifying anti-rheumatic drugs" (bDMARDs), aber auch im Hinblick auf eine frühe Diagnosestellung nachgewiesen $[3,5,6]$.

Die Entwicklung und Festlegung von einfach messbaren Qualitätsstandards (QS) kann helfen, Lücken in der Versorgung zu schließen und die Versorgung zu verbessern [7]. Die Entwicklung von nationalen und internationalen Leitlinien (LL) und Empfehlungen gilt bereits als großer Schritt in Richtung einer soliden Evidenzbasis für therapeutische Entscheidungen in der Medizin allgemein, aber auch in der Rheumatologie im Besonderen [8]. Die Gemeinsamkeit von LL und QS besteht darin, dass beide durch Expert*innen in meist aufwendigen Prozessen entwickelt werden. Der Unterschied zwischen LL und QS dagegen ist, dass Erstere evidenzbasierte Empfehlungen für das Management von Erkrankungen, z. T. auch in unterschiedlichen Stadien, hervorbringen, während Letztere sich durch den Einsatz von messbaren Qualitätskonstrukten auszeichnen, indem Instrumente definiert werden, mit denen Versorgungsqualität in den Domänen Strukturqualität, Prozessqualität und Ergebnisqualität gemessen werden kann [9].

Für einige Erkrankungen aus dem rheumatologischen Erkrankungskreis, wie z.B. der rheumatoiden Arthritis, 
Hier steht eine Anzeige.

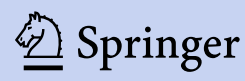




\begin{tabular}{|c|c|c|c|}
\hline $\begin{array}{l}\text { Qualitäts- } \\
\text { standard }\end{array}$ & Thema & $\begin{array}{l}\text { Zustimmung } \\
\text { ja/nein }\end{array}$ & $\begin{array}{l}\text { Grad der Zustimmung (NRS } \\
0-10), M W \pm S D\end{array}$ \\
\hline QS 1 & Überweisung & $15 / 0$ & $8,9 \pm 1,6$ \\
\hline QS 2 & Zeit bis zum Termin & $15 / 0$ & $9,6 \pm 0,7$ \\
\hline QS 3 & Diagnostische Abklärung & $15 / 0$ & $9,7 \pm 0,5$ \\
\hline QS 4 & Monitoring & $15 / 0$ & $9,7 \pm 0,5$ \\
\hline QS 5 & $\begin{array}{l}\text { Leitliniengerechte Therapiees- } \\
\text { kalation }\end{array}$ & $13 / 0$ & $9,8 \pm 0,4$ \\
\hline QS 6 & $\begin{array}{l}\text { Nicht-pharmakologische Be- } \\
\text { handlung }\end{array}$ & $15 / 0$ & $9,7 \pm 0,6$ \\
\hline QS 7 & Patient*innenschulung & $15 / 0$ & $8,8 \pm 1,5$ \\
\hline QS 8 & Kurzfristiger Termin & $15 / 0$ & $7,8 \pm 2,4$ \\
\hline QS 9 & Jährliche Beurteilung & $15 / 0$ & $9,1 \pm 1,3$ \\
\hline
\end{tabular}

sind international bereits Instrumente zur Qualitätsbeurteilung vorgeschlagen worden [10-13]; für axSpA war das bisher nicht der Fall. Die Assessment of Spondyloarthritis International Society (ASAS) hatte es sich daher zur Aufgabe gemacht, einfach einsetzbare international akzeptierte QS für Patient*innen mit axSpA zu entwickeln. Dabei orientierte sich die Organisation an der Vorgehensweise des vom National Institute for Health and Care Excellence (NICE) entwickelten Ansatz zur Entwicklung von QS $[14,15]$. Dieser zeichnete sich durch die Beteiligung aller relevanten Interessengruppen unter Einbeziehung der vorliegenden wissenschaftlichen Evidenz für die Entwicklung von QS aus.

Grundsätzlich bestehen QS aus 2 Komponenten: einer Qualitätsaussage und einer Qualitätsmessung. Die Qualitätsaussage, auf die sich eine Expert ${ }^{\star}$ innenkommission geeinigt hat, definiert die gewünschte Versorgungsqualität auf Basis einer prägnanten und potenziell messbaren Aussage, die mit vorhandenen evidenzbasierten Leitlinien kompatibel ist bzw. sein sollte. Hierbei geht es darum, einen Versorgungsbereich $\mathrm{zu}$ identifizieren, der sowohl relevant als auch erreichbar und messbar ist, um auf dieser Basis die gewünschte Versorgungsqualität zu definieren. Jeder Aussage wird eine Rationale zugrunde gelegt, die auf wissenschaftlicher Evidenz, verfügbaren LL, bestehenden Empfehlungen und der Einschätzung der Expert*innen auf mögliche Auswirkungen der QS auf Zielgruppen (Dienstleister, öffentliches Gesundheits- wesen und Soziales, Patient*innen etc.) beruht. Ob die in der Qualitätsaussage gewünschte Versorgungsqualität erreicht wurde, kann durch Qualitätsmessinstrumente quantifiziert werden. Die dafür erforderlichen Instrumente sind für die etablierten Qualitätsdomänen Struktur-, Prozess- und Ergebnisqualität unterschiedlich. Um ein besseres Ergebnis, d. h. eine bessere Versorgungsqualität zu erreichen, sind eine solide Struktur und gut durchdachte Prozesse erforderlich. Für die Instrumente zur Messung der Qualität muss jeweils definiert werden, welche Größe im Zähler und welche im Nenner steht [15-17].

Im Auftrag der Deutschen Gesellschaft für Rheumatologie (DGRh) und in Anlehnung an die von der ASAS publizierten Vorgabe sowie auf Basis der deutschen S3-Leitlinie war das Ziel dieses Projektes, die international entwickelten QS an nationale Gegebenheiten anzupassen und QS für axSpA-Patient*innen in Deutschland zu entwickeln.

\section{Methoden}

Zu Beginn des Projektes im September 2019 wurden eine Steuerungsgruppe (KA, JB, UK, H-JL, H-ML, MR, MS, HS-K) und eine Arbeitsgruppe (AG) mit ausgewählten Expert*innen gebildet. Die Steuerungsgruppe entschied initial über die zu verwendende Methodik sowie die Beteiligung verschiedener Interessengruppen. Die AG setzte sich aus Mitgliedern der Steuerungsgruppe sowie verschiedenen Interessengruppen (stationär und ambulant tätige Rheuma- tolog*innen und einem Patientenvertreter der Deutschen Vereinigung Morbus Bechterew e.V. [DVMB]) zusammen.

Die internationalen von ASAS publizierten QS für axSpA wurden als Grundlage für die Entwicklung akzeptiert und die Adaptation für den deutschsprachigen Raum geplant, wobei angesichts der aktuellen Pandemie keine Expert ${ }^{\star}$ innen aus Österreich bzw. der Schweiz hinzugezogen wurden.

Die geplante Methodik sah zunächst die Übersetzung der englischen Originalfassung in eine deutsche Version durch Mitglieder der AG auf Grundlage eines Vorschlags von JB und UK vor. Im Anschluss daran hatten die Mitglieder der AG die Möglichkeit, diesen Übersetzungsvorschlag zu kommentieren. In einem virtuellen Treffen wurden dann Änderungswünsche diskutiert, und anschließend wurde die finale Übersetzung konsentiert. Vor dem Hintergrund der methodischen Vorgaben führten die Mitglieder der AG eine inhaltliche Prüfung der Empfehlungen durch, passten diese an die deutsche Versorgungssituation an und identifizierten mögliche Hinderungsfaktoren für die Verbreitung und Umsetzung der QS in Deutschland. Abschließend wurden alle Mitglieder der AG gebeten, zu jedem einzelnen QS ihre Zustimmung oder Ablehnung zu bekunden sowie den Grad ihrer Zustimmung mithilfe einer numerischen Rating-Skala (NRS) mitzuteilen (Grad $0=$ stimme nicht $\mathrm{zu}$, Grad $10=$ stimme voll $\mathrm{zu}$ ).

\section{Ergebnisse}

Der erste Übersetzungsvorschlag (JB) wurde im Sommer 2020 in einem ersten Umlaufverfahren diskutiert und in einem wegen der Corona-Pandemie virtuell durchgeführten Treffen im September 2020 erneut kommentiert und letztlich konsentiert. Im Rahmen des Abstimmungsprozesses wurde zunächst die englische Version kritisch geprüft und befunden, dass diese nicht 1:1 auf Deutschland übertragen werden kann. Entsprechend wurden einige Formulierungen und hier insbesondere der QS 5 abgeändert und neu formuliert. Dieser QS weicht nun deutlich vom 
englischsprachigen Original der ASAS ab.

Insgesamt nahmen 15 AG-Mitglieder an der Abstimmung der QS während des virtuellen Meetings teil. An der anschließend durchgeführten OnlineUmfrage nahmen 10 der 15 eingeladenen Mitglieder teil und bewerteten den Grad der Zustimmung (NRS 0-10) jedes einzelnen Qualitätsstandards (• Tab. 1). Hierbei wurde ein minimaler Grad der Zustimmung bei QS 8 (Kurzfristiger Termin) mit 7,8 $\pm 2,4$ und ein maximaler Grad der Zustimmung bei QS 5 (Leitliniengerechte Therapieeskalation) mit 9,8 $\pm 0,4$ erreicht. Im Durchschnitt lag der Grad der Zustimmung aller QS bei $9,3 \pm 0,7$.

\section{Übersetzung der einzelnen QS}

Es wurden insgesamt 9 QS der internationalen Version übersetzt. Vor der Abstimmung der einzelnen QS diskutierten die AG-Teilnehmer*innen den Gebrauch einer geschlechtsneutralen Sprache. Im letztlich durchgeführten Abstimmungsprozess stimmten 14 von 15 Mitgliedern für die Verwendung einer geschlechtsneutralen Sprache in Form eines Gendersternchens (1 Mitglied enthielt sich). Kritisch angemerkt wurde, dass sich die Lesbarkeit des Manuskripts durch die Gendersternchen leider deutlich erschwert.

Im Folgenden wird auf die einzelnen QS eingegangen, und die Diskussionen und Kommentare während der Abstimmung werden beschrieben und erläutert.

Die Ausformulierung der QS mit Rationale und Messwerten zeigen - Tab. 2, $3,4,5,6,7,8,9$ und 10 .

\section{QS 1: Überweisung}

Zunächst wurde über die Änderung des Begriffes „Überweisung“ in „Zuweisung“ diskutiert, da der Begriff der „Überweisung" ein kassenärztlicher Terminus ist. Mehrheitlich wurde sich dann jedoch für den Begriff „Überweisung“ entschieden.

Im Unterschied zu den internationalen ASAS-QS wurde bei der deutschen Übersetzung auf den Begriff „lokal“ bei der Kategorie Struktur „Vorhaltung [lokaler] Maßnahmen und Strukturen [... ]“

Z Rheumatol 2022 $\cdot 81: 730-743$ https://doi.org/10.1007/s00393-021-01019-x

(c) Der/die Autor(en) 2021

U. Kiltz • V. Buschhorn-Milberger · K. Albrecht · H.-J. Lakomek · H.-M. Lorenz · M. Rudwaleit • M. Schneider · H. Schulze-Koops · X. Baraliakos - F. Behrens · J. Brandt-Jürgens · H. Haibel · L. Hammel · K. Karberg · H. Kellner · D. Krause - U. Lange · E. Märker-Herrmann ·

D. Poddubnyy $\cdot$ J. Sieper $\cdot$ U. Syrbe $\cdot$ J. Braun

\section{Entwicklung von Qualitätsstandards für Patient*innen mit axialer Spondyloarthritis zum Einsatz in Deutschland}

\section{Zusammenfassung}

Qualitätsstandards (QS) sind messbare Konstrukte, die helfen sollen, Versorgungslücken quantitativ zu erfassen, um langfristig die Versorgungsqualität zu verbessern. Die Assessment of SpondyloArthritis International Society (ASAS) hat kürzlich erstmals internationale QS für das Management von Patient*innen mit axialer Spondyloarthritis (axSpA) konsentiert und veröffentlicht. Die Deutsche Gesellschaft für Rheumatologie (DGRh) hat daraufhin beschlossen, diese Standards durch eine Gruppe von Expert*innen aus unterschiedlichen Versorgungsbereichen zu übersetzen, zu prüfen und ggf. zu übernehmen. Vor diesem Hintergrund wurden erstmals nationale $Q S$ für das Management von Patient*innen mit axSpA für Deutschland entwickelt. Hierbei wurde v. a. auf Machbarkeit und Praxisrelevanz geachtet. Letztlich wurden 9 QS definiert, mit denen die Qualität der Versorgung in Deutschland gemessen und verbessert werden kann bzw. soll.

Schlüsselwörter

Axiale Spondyloarthritis · Ankylosierende Spondylitis - Versorgungsqualität . Qualitätsstandards · Versorgungslücken

\section{Development of quality standards for patients with axial spondyloarthritis for use in Germany}

\section{Abstract}

Quality standards (QS) are measurable constructs designed to quantify gaps in care and subsequently to improve quality of care. The Assessment of SpondyloArthritis International Society (ASAS) recently generated and published international QS for the management of patients with axial spondyloarthritis (axSpA) for the first time. The German Society of Rheumatology (DGRh) then decided to translate, review and possibly adopt these standards by a group of experts from different care settings. Against this background, national QS for the management of patients with axSpA for Germany were developed for the first time. The main focus was on feasibility and practical relevance. Ultimately, nine QS were defined with which the quality of care in Germany can and should be measured and improved.

\section{Keywords}

Axial spondyloarthritis - Ankylosing spondylitis - Quality of care · Quality standards · Care gaps verzichtet, da lokal zu eng definiert ist und in einigen Teilen Deutschlands eine überregionale oder sektorale Versorgung stattfindet, welche durch den Begriff „lokal“ nicht abgebildet wird. Ebenfalls angemerkt wurde, dass geeignete Maßnahmen und Strukturen besser grundsätzlich vorhanden und nicht ortsbezogen begrenzt sein sollten (• Tab. 2).

Nach erfolgten Änderungen stimmten 15 der 15 Mitglieder für die finale Übersetzung.

\section{QS 2: Zeit bis zum Termin bei Rheumatolog*innen}

Es wurde die Frage diskutiert, was in den internationalen ASAS QS mit „specialist" gemeint ist. Während in anderen Ländern zum Teil auch in verschiedenen Fachrichtungen tätige Ärzt ${ }^{\star}$ innen in der Versorgung von Patient*innen mit rheumatischen Erkrankungen tätig sind, ist in Deutschland vorwiegend der $^{\star}$ die internistische Rheumatolog*in, meist in Kooperation mit dem $^{\star}$ der Hausarzt ${ }^{\star a ̈ r z t i n, ~}$ für die Versorgung verantwortlich. 


\begin{tabular}{|c|c|c|c|c|c|}
\hline Domäne & Standard & Rationale & $\begin{array}{l}\text { Qualitätsmessung, Kate- } \\
\text { gorie Struktur }\end{array}$ & $\begin{array}{l}\text { Qualitätsmessung, Ka- } \\
\text { tegorie Prozess, Zähler }\end{array}$ & $\begin{array}{l}\text { Qualitäts- } \\
\text { messung, } \\
\text { Kategorie } \\
\text { Prozess, Nen- } \\
\text { ner }\end{array}$ \\
\hline $\begin{array}{l}\text { Überwei- } \\
\text { sung }\end{array}$ & $\begin{array}{l}\text { Patient*innen mit } \\
\text { Verdacht auf axSpA } \\
\text { werden innerhalb } \\
\text { von } 3 \text { Werktagen } \\
\text { zur diagnostischen } \\
\text { Abklärung an ei- } \\
\text { ne*n Rheumato- } \\
\log ^{*} \text { in überwiesen }\end{array}$ & $\begin{array}{l}\text { Eine axSpA wird von Nicht-Rheuma- } \\
\text { tolog*innen häufig nicht erkannt, was } \\
\text { zu erheblichen Verzögerungen bei } \\
\text { Diagnose und Behandlung führt. Es } \\
\text { gibt keinen diagnostischen Test, der } \\
\text { allein eine ausreichende Sensitivität } \\
\text { oder Spezifität für die Diagnose axSpA } \\
\text { aufweist. Die ASAS-Empfehlungen } \\
\text { enthalten Kriterien für die Entschei- } \\
\text { dung zu einer zeitnahen Überweisung } \\
\text { von Patient*innen mit Verdacht auf } \\
\text { axSpA in die Rheumatologie. Die auf } \\
\text { Expert*innenmeinung basierende } \\
\text { Wahl des Zeitraums von } 3 \text { Arbeitsta- } \\
\text { gen soll eine sofortige Überweisung } \\
\text { (z. B. durch Hausärzt*innen) bewirken }\end{array}$ & $\begin{array}{l}\text { Vorhaltung von Maßnahmen } \\
\text { und Strukturen (einschließ- } \\
\text { lich Vorkehrungen zur Sen- } \\
\text { sibilisierung für Anzeichen } \\
\text { und Symptome von axSpA) } \\
\text { und vereinbarte Überwei- } \\
\text { sungsverfahren, um sicher- } \\
\text { zustellen, dass Patient*innen } \\
\text { mit Verdacht auf axSpA in- } \\
\text { nerhalb von } 3 \text { Werktagen } \\
\text { an eine*n Rheumatolog*in } \\
\text { überwiesen werden }\end{array}$ & $\begin{array}{l}\text { Die Anzahl der Pa- } \\
\text { tient*innen mit Verdacht } \\
\text { auf axSpA, die innerhalb } \\
\text { von } 3 \text { Werktagen an Rheu- } \\
\text { matolog*innen überwie- } \\
\text { sen wurden. Die } 3 \text { Tage } \\
\text { werden zum einen an der } \\
\text { Dokumentation der Ver- } \\
\text { dachtsdiagnose und zum } \\
\text { anderen an der Ausstellung } \\
\text { des Überweisungsscheins } \\
\text { gemessen }\end{array}$ & $\begin{array}{l}\text { Die Anzahl der } \\
\text { Patient*innen } \\
\text { mit Verdacht } \\
\text { auf axSpA }\end{array}$ \\
\hline
\end{tabular}

Damit wurde von der AG hinsichtlich der ärztlichen Zuständigkeit für das Management von Patient ${ }^{*}$ innen mit axSpA klar definiert, dass bei allen QS grundsätzlich der $^{\star}$ die internistische Rheumatolog*in als verantwortliche ${ }^{\star} r$ Facharzt*ärztin gemeint ist. Grundlage hierfür sind die für eine adäquate Patient ${ }^{*}$ innenversorgung erforderliche internistische Facharztausbildung mit fundierter Kenntnis über die differenzialdiagnostische Abklärung sowie ein breites Wissen über medikamentöse Therapieprinzipien und deren Management im Langzeitverlauf auch hinsichtlich unerwünschter Wirkungen und Komplikationen.

Daher wurde die Übersetzung in „Zeit [...] bei Rheumatolog*innen“" anstatt "Zeit [...] bei Spezialist ${ }^{*}$ innen $^{\text {“ }}$ abgeändert (- Tab. 3).

Nach erfolgten Änderungen stimmten 15 der 15 Mitglieder für die finale Übersetzung.

\section{QS 3: Diagnostische Abklärung durch Rheumatolog*innen}

Die ursprüngliche englische Benennung „assessment" wurde kritisch diskutiert vor dem Hintergrund, dass die erfolgten Untersuchungen und Evaluierungen vom Kontext abhängen und somit nicht per se ein reines Assessment darstellen. Es wurde entschieden, dass mit dem Begriff „diagnostische Abklärung“ den verschiedenen klinischen, radiologischen und laborchemischen Untersuchungen besser Rechnung getragen wird.

Ebenfalls diskutiert wurde, ob das „langfristige Outcome“ oder der „langfristige Krankheitsverlauf “ betrachtet werden soll. Da das „langfristige Outcome“ im engeren Sinne einen in der Zukunft liegenden Status der Erkrankung und nicht den Verlauf der Krankheit meint, wurde entschieden, den Begriff „Krankheitsverlauf“ zu nehmen (- Tab. 4).

Nach erfolgten Änderungen stimmten 15 der 15 Mitglieder für die finale Übersetzung.

\section{QS 4: Monitoring}

Grundsätzlich sollten die mit der Festlegung eines QS beabsichtigten Ziele nicht nur für die Versorgung wünschenswert, sondern auch erreichbar sein. Daher wurde in diesem QS ein Zeitraum von 6 Monaten gewählt. Dies wurde von einigen Mitgliedern allerdings eher als zu lang als zu kurz eingestuft.

In Zukunft wäre die mögliche Delegation einiger ärztlicher Aufgaben z.B. an eine rheumatologische Fachassistenz wünschenswert, daher wurde in diesem QS die entsprechende Formulierung gewählt, dass Patient*innen „[...] unter Supervision einer ${ }^{\star}$ s Rheumatolog*in $[\ldots]^{\star}$ regelmäßig evaluiert werden, anstatt diesen QS lediglich auf den Tätigkeitsbereich der $^{*}$ s Rheumatolog*in zu begrenzen (• Tab. 5).

Nach erfolgten Änderungen stimmten 15 der 15 Mitglieder für die finale Übersetzung.

\section{QS 5: Leitliniengerechte Therapieeskalation}

Generell wurde angemerkt, dass im Gegensatz zur internationalen Situation, die dadurch gekennzeichnet ist, dass in einigen Ländern bei Patient ${ }^{*}$ innen mit axSpA relativ wenig bDMARDs verordnet werden, die Versorgungslücke in Deutschland durch eine unzureichende leitliniengerechte Therapie insofern bedingt ist, als dass es Anhaltspunkte für eine Unterversorgung dieser Patient ${ }^{*}$ innen mit Physiotherapie gibt (hierauf wird im QS 6 näher eingegangen) $[18,19]$. Für eine qualitativ mangelhafte Versorgung mit NSAR gibt es insgesamt keine sicheren Hinweise [20]. Auch die Möglichkeit einer medikamentösen Überversorgung wurde thematisiert. Einige AG-Mitglieder bemängelten in diesem Zusammenhang, dass die aktuellen QS die Möglichkeit einer Deeskalation der Therapie nur unzureichend adressieren.

Prinzipiell wurde in diesem Zusammenhang betont, dass eine Verordnung von Medikamenten nicht nur medizi- 
Hier steht eine Anzeige.

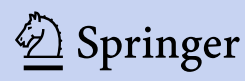




\begin{tabular}{|c|c|c|c|c|c|}
\hline Domäne & Standard & Rationale & $\begin{array}{l}\text { Qualitätsmessung, } \\
\text { Kategorie Struktur }\end{array}$ & $\begin{array}{l}\text { Qualitätsmessung, } \\
\text { Kategorie Prozess, } \\
\text { Zähler }\end{array}$ & $\begin{array}{l}\text { Qualitäts- } \\
\text { messung, } \\
\text { Kategorie } \\
\text { Prozess, Nen- } \\
\text { ner }\end{array}$ \\
\hline $\begin{array}{l}\text { Zeit bis } \\
\text { zum Ter- } \\
\text { min bei } \\
\text { Rheu- } \\
\text { mato- } \\
\text { log*innen }\end{array}$ & $\begin{array}{l}\text { Patient*innen } \\
\text { mit Verdacht auf } \\
\text { axSpA werden } \\
\text { innerhalb von } \\
3 \text { Wochen nach } \\
\text { der Überweisung } \\
\text { von einem*einer } \\
\text { Rheumatolog*in } \\
\text { untersucht }\end{array}$ & $\begin{array}{l}\text { Eine schnelle Überweisung von Patient*innen } \\
\text { mit Verdacht auf axSpA ist wichtig, um Verzö- } \\
\text { gerungen bei der Diagnosestellung zu vermei- } \\
\text { den und die Wahrscheinlichkeit eines frühen } \\
\text { Behandlungsbeginns zu erhöhen. Ein*e Rheu- } \\
\text { matolog*in (der Terminus bezieht sich auf das } \\
\text { gesamte rheumatologische Team, bestehend } \\
\text { aus Ärzt*innen, Pflegepersonal und anderen } \\
\text { medizinischen Fachkräften) ist in der Lage, } \\
\text { axiale und periphere Manifestationen sowie ex- } \\
\text { traartikuläre Manifestationen und Komorbiditä- } \\
\text { ten zu identifizieren. Angesichts der potenziell } \\
\text { nachteiligen Auswirkungen einer verspäteten } \\
\text { Diagnose benötigen Patient*innen mit diesen } \\
\text { Symptomen und Anzeichen einen ersten Ter- } \\
\text { min innerhalb von } 3 \text { Wochen. Der Zeitrahmen } \\
\text { basiert auf Expert*innenmeinung, um die Ver- } \\
\text { gabe von zeitnahen Terminen anzuregen. Die } \\
\text { Frist von } 3 \text { Wochen bezieht sich auf den ersten } \\
\text { Termin. Zusätzliche Untersuchungen, die für } \\
\text { die Diagnosefindung erforderlich sind, können } \\
\text { auch nach dem ersten Termin erfolgen }\end{array}$ & $\begin{array}{l}\text { Vorhaltung von Maß- } \\
\text { nahmen und Strukturen, } \\
\text { einschließlich einer aus- } \\
\text { reichenden Anzahl von } \\
\text { Rheumatolog*innen, um } \\
\text { sicherzustellen, dass Pa- } \\
\text { tient*innen mit Verdacht } \\
\text { auf axSpA innerhalb von } \\
3 \text { Wochen nach der Über- } \\
\text { weisung von einem*einer } \\
\text { Rheumatolog*in gesehen } \\
\text { werden }\end{array}$ & $\begin{array}{l}\text { Die Anzahl der Pa- } \\
\text { tient*innen mit Ver- } \\
\text { dacht auf axSpA, die } \\
\text { von einem*einer Rheu- } \\
\text { matolog*in innerhalb } \\
\text { von } 3 \text { Wochen nach der } \\
\text { Überweisung unter- } \\
\text { sucht werden }\end{array}$ & $\begin{array}{l}\text { Die Anzahl der } \\
\text { Patient*innen } \\
\text { mit Verdacht } \\
\text { auf axSpA, } \\
\text { die an ei- } \\
\text { ne*n Rheu- } \\
\text { matolog*in } \\
\text { überwiesen } \\
\text { wurden }\end{array}$ \\
\hline
\end{tabular}

Tab.4 Qualitätsstandard 3: Diagnostische Abklärung durch Rheumatolog*innen

\begin{tabular}{|c|c|c|c|c|c|}
\hline Domäne & Standard & Rationale & $\begin{array}{l}\text { Qualitätsmessung, Kate- } \\
\text { gorie Struktur }\end{array}$ & $\begin{array}{l}\text { Qualitätsmessung, Ka- } \\
\text { tegorie Prozess, Zähler }\end{array}$ & $\begin{array}{l}\text { Qualitäts- } \\
\text { messung, } \\
\text { Kategorie } \\
\text { Prozess, Nen- } \\
\text { ner }\end{array}$ \\
\hline $\begin{array}{l}\text { Diagnos- } \\
\text { tische Ab- } \\
\text { klärung } \\
\text { durch } \\
\text { Rheumato- } \\
\text { log*innen }^{*}\end{array}$ & $\begin{array}{l}\text { Bei Verdacht auf } \\
\text { axSpA wird die rheu- } \\
\text { matologische Abklä- } \\
\text { rung innerhalb von } \\
2 \text { Monaten abge- } \\
\text { schlossen }\end{array}$ & $\begin{array}{l}\text { Eine rechtzeitige Abklärung durch ei- } \\
\text { ne*n Rheumatolog*in ist notwendig, } \\
\text { um eine korrekte Diagnose stellen zu } \\
\text { können. Durch eine frühzeitige Dia- } \\
\text { gnose wird der langfristige Krankheits- } \\
\text { verlauf verbessert und die Lebensqua- } \\
\text { lität gesteigert. Die diagnostische Ab- } \\
\text { klärung beinhaltet die Identifizierung } \\
\text { von SpA-Symptomen, Laborparameter } \\
\text { und eine entsprechende Bildgebung. } \\
\text { Diese sollte innerhalb von } 2 \text { Mona- } \\
\text { ten nach der ersten Vorstellung bei } \\
\text { dem*der Rheumatolog*in abgeschlos- } \\
\text { sen sein }\end{array}$ & $\begin{array}{l}\text { Vorhaltung von Maßnahmen } \\
\text { und Strukturen, einschließ- } \\
\text { lich einer ausreichenden } \\
\text { Anzahl von Rheumato- } \\
\text { log*innen und Einrichtun- } \\
\text { gen sowie der Zugang zu } \\
\text { diesen Einrichtungen in- } \\
\text { nerhalb des vorgegebenen } \\
\text { Zeitrahmens, um sicherzu- } \\
\text { stellen, dass Patient*innen } \\
\text { mit Verdacht auf axSpA in- } \\
\text { nerhalb von } 2 \text { Monaten nach } \\
\text { der ersten Vorstellung durch } \\
\text { eine*n Rheumatolog*in eine } \\
\text { diagnostische Abklärung } \\
\text { erhalten }\end{array}$ & $\begin{array}{l}\text { Die Anzahl der Pa- } \\
\text { tient*innen mit Verdacht } \\
\text { auf axSpA, bei denen eine } \\
\text { diagnostische Abklärung } \\
\text { innerhalb von } 2 \text { Monaten } \\
\text { nach der ersten Vorstellung } \\
\text { abgeschlossen wurde }\end{array}$ & $\begin{array}{l}\text { Die Anzahl der } \\
\text { Patient*innen } \\
\text { mit Verdacht } \\
\text { auf axSpA, die } \\
\text { der*die Rheu- } \\
\text { matolog*in } \\
\text { vor mehr als } \\
2 \text { Monaten } \\
\text { erstmalig ge- } \\
\text { sehen hat }\end{array}$ \\
\hline
\end{tabular}

nisch, sondern auch im Sinne eines adäquaten wirtschaftlichen Handelns vertretbar sein sollte. Dazu gehört auch, dass die Verordnung eines bDMARDs beivorliegender hoher Krankheitsaktivität nicht nur zu einer Versorgungsverbesserung für den ${ }^{\star}$ die jeweilige ${ }^{\star} n$ Patient ${ }^{*}$ in führen kann, sondern durch die Vermeidung einer Hospitalisierung auch durchaus wirtschaftlich sinnvoll ist, weil damit Kosten für stationäre Versorgung und Arbeitsunfähigkeit eingespart werden können [21].

Zudem wurde die Begrifflichkeit „konventionelle Therapie“ diskutiert. Im internationalen rheumatologischen Wortschatz fallen unter den Begriff „conventional synthetic“ (cs)DMARDs gängige Medikamente der Basisversorgung wie MTX, Leflunomid oder Sulfasalazin, für die eine krankheitsmodifizierende Wirkung im Sinne einer Hemmung der Röntgenprogression nachgewiesen wurde. Das gilt allerdings eigentlich nur für die rheumatoide Arthritis, bzw. wurde bei Patient*innen mit dieser Erkrankung gezeigt. Der Terminus wurde für axSpA übernommen, obwohl eine solche Wirkung bei dieser Erkrankung weder nachgewiesen wurde noch anzunehmen 


\begin{tabular}{|c|c|c|c|c|c|}
\hline Domäne & Standard & Rationale & $\begin{array}{l}\text { Qualitätsmessung, } \\
\text { Kategorie Struktur }\end{array}$ & $\begin{array}{l}\text { Qualitätsmessung, } \\
\text { Kategorie Prozess, } \\
\text { Zähler }\end{array}$ & $\begin{array}{l}\text { Qualitäts- } \\
\text { messung, } \\
\text { Kategorie } \\
\text { Prozess, Nen- } \\
\text { ner }\end{array}$ \\
\hline Monitoring & $\begin{array}{l}\text { Die Krankheits- } \\
\text { aktivität von } \\
\text { Patient*innen } \\
\text { mit axSpA wird } \\
\text { unter Supervision } \\
\text { durch Rheuma- } \\
\text { tolog*innen mit } \\
\text { validierten Mess- } \\
\text { instrumenten } \\
\text { mindestens alle } \\
6 \text { Monate evalu- } \\
\text { iert }\end{array}$ & $\begin{array}{l}\text { Die Beurteilung der Krankheitsaktivität ist we- } \\
\text { gen des Zusammenhangs zwischen klinischer } \\
\text { Krankheitsaktivität und Syndesmophyten- } \\
\text { bildung sowie zwischen Krankheitsaktivität, } \\
\text { Funktionsfähigkeit und gesundheitsbezo- } \\
\text { gener Lebensqualität von Bedeutung. Die } \\
\text { Überwachung der Krankheitsaktivität durch } \\
\text { eine*n Rheumatolog*in (der Terminus bezieht } \\
\text { sich auf das gesamte rheumatologische Team, } \\
\text { bestehend aus Ärzt*innen, Pflegepersonal, } \\
\text { rheumatologischer Fachassistenz und ande- } \\
\text { ren medizinischen Fachkräften) ist wegen der } \\
\text { vielfältigen und mehrdeutigen klinischen Sym- } \\
\text { ptome der Krankheitsaktivität wie Schmerzen } \\
\text { und Behinderungen erforderlich. Eine Bewer- } \\
\text { tung der Krankheitsaktivität mittels ASDAS wird } \\
\text { empfohlen. Durch den regelmäßigen Einsatz } \\
\text { der Messinstrumente wird sichergestellt, dass } \\
\text { die Behandlung von Patient*innen mit axSpA } \\
\text { bei Bedarf angepasst werden kann }\end{array}$ & $\begin{array}{l}\text { Vorhaltung von Maß- } \\
\text { nahmen und Strukturen, } \\
\text { um sicherzustellen, dass } \\
\text { Patient*innen mit axSpA } \\
\text { mindestens alle } 6 \text { Monate } \\
\text { mit validierten Messin- } \\
\text { strumenten evaluiert } \\
\text { werden }\end{array}$ & $\begin{array}{l}\text { Die Anzahl der Pa- } \\
\text { tient*innen, bei denen } \\
\text { vor mehr als } 6 \text { Monaten } \\
\text { eine axSpA diagnos- } \\
\text { tiziert wurde und bei } \\
\text { denen die Krankheitsak- } \\
\text { tivität mindestens alle } \\
6 \text { Monate mit validier- } \\
\text { ten Messinstrumenten } \\
\text { überwacht wurde }\end{array}$ & $\begin{array}{l}\text { Die Anzahl der } \\
\text { Patient*innen, } \\
\text { bei denen } \\
\text { vor mehr als } \\
6 \text { Monaten } \\
\text { eine axSpA } \\
\text { diagnostiziert } \\
\text { wurde }\end{array}$ \\
\hline
\end{tabular}

Tab.6 Qualitätsstandard 5: Leitliniengerechte Therapieeskalation

\begin{tabular}{|c|c|c|c|c|c|}
\hline Domäne & Standard & Rationale & $\begin{array}{l}\text { Qualitätsmessung, } \\
\text { Kategorie Struktur }\end{array}$ & $\begin{array}{l}\text { Qualitätsmessung, } \\
\text { Kategorie Prozess, } \\
\text { Zähler }\end{array}$ & $\begin{array}{l}\text { Qualitäts- } \\
\text { messung, } \\
\text { Kategorie } \\
\text { Prozess, Nen- } \\
\text { ner }\end{array}$ \\
\hline $\begin{array}{l}\text { Leitlinien- } \\
\text { gerechte } \\
\text { Therapie- } \\
\text { eskalation }\end{array}$ & $\begin{array}{l}\text { Bei Patient*innen } \\
\text { mit axSpA und } \\
\text { hoher Krankheits- } \\
\text { aktivität ist das } \\
\text { zentrale Ziel, eine } \\
\text { Remission durch } \\
\text { Therapieeskalati- } \\
\text { on entsprechend } \\
\text { der aktuellen } \\
\text { S3-Leitlinie zu } \\
\text { erreichen }\end{array}$ & $\begin{array}{l}\text { Eine Therapieeskalation ist wichtig, um die } \\
\text { Krankheitsaktivität zu kontrollieren. Dies erfolgt } \\
\text { mit dem Ziel, idealerweise eine Remission oder } \\
\text { einen Zustand niedriger Krankheitsaktivität } \\
\text { zu erreichen, um den Einfluss der Erkrankung } \\
\text { auf die Funktionsfähigkeit und den Alltag der } \\
\text { Patient*innen zu minimieren. Patient*innen, } \\
\text { die trotz konventioneller Therapie eine ho- } \\
\text { he Krankheitsaktivität haben, sollten mit ih- } \\
\text { rem*ihrer Rheumatolog*in die verschiedenen }^{*} \text { Therapiemöglichkeiten besprechen. Die ak- } \\
\text { tuelle S3-Leitlinie für das Management von } \\
\text { Patient*innen mit axSpA enthält Kriterien für } \\
\text { den Einsatz von medikamentösen und nicht- } \\
\text { medikamentösen Therapien bei Patient*innen } \\
\text { mit hoher Krankheitsaktivität. Die Therapie- } \\
\text { entscheidung sollte auf einer gemeinsamen } \\
\text { Entscheidung zwischen Patient*innen und } \\
\text { Rheumatolog*innen beruhen }\end{array}$ & $\begin{array}{l}\text { Vorhaltung von Maß- } \\
\text { nahmen und Strukturen, } \\
\text { um sicherzustellen, dass } \\
\text { Patient*innen mit axSpA } \\
\text { und hoher Krankheitsakti- } \\
\text { vität gemäß der aktuellen } \\
\text { S3-Leitlinie behandelt } \\
\text { werden können, um die } \\
\text { Chance auf Remission } \\
\text { oder geringe Krankheits- } \\
\text { aktivität zu maximieren }\end{array}$ & $\begin{array}{l}\text { Die Anzahl der Pa- } \\
\text { tient*innen mit axSpA } \\
\text { und hoher Krankheits- } \\
\text { aktivität, bei denen } \\
\text { eine Therapieeskalation } \\
\text { gemäß der aktuellen } \\
\text { S3-Leitlinie durchge- } \\
\text { führt wurde }\end{array}$ & $\begin{array}{l}\text { Die Anzahl der } \\
\text { Patient*innen } \\
\text { mit axSpA und } \\
\text { hoher Krank- } \\
\text { heitsaktivität }\end{array}$ \\
\hline
\end{tabular}

ist. Die Biologika laufen unter dem Begriff „biological“ (b)DMARDs, wobei es neuerdings auch "targeted synthetic“ (ts)DMARDs wie die Januskinaseinhibitoren gibt, der erste ist inzwischen zugelassen.

Kritisch wurde bemerkt, dass dieser QS nicht das Problemfeld des Zuganges zur Therapie beleuchtet. Explizit wurden hier Patient*innen ohne Krankenversicherung angeführt, welche zwar zum aktuellen Zeitpunkt in Deutschland ein vergleichsweise kleines Problem darstellen, was jedoch in den nächsten Jahren zunehmend an Bedeutung gewinnen könnte. Das Statistische Bundesamt hat kürzlich Zahlen veröffentlicht, wonach im Jahr 2019 immerhin 143.000 Menschen, al- so knapp 0,1\% der Bevölkerung, nicht krankenversichert waren. Im Vergleich dazu waren es im Jahr 2015 nur rund 79.000 ([22]; - Tab. 6).

Nach erfolgten Änderungen stimmten die 13 noch anwesenden Mitglieder für die finale Übersetzung. Da dieses QS als Letztes abgestimmt wurde, konnten 


\begin{tabular}{|c|c|c|c|c|c|}
\hline Domäne & Standard & Rationale & $\begin{array}{l}\text { Qualitätsmes- } \\
\text { sung, Kategorie } \\
\text { Struktur }\end{array}$ & $\begin{array}{l}\text { Qualitätsmes- } \\
\text { sung, Kate- } \\
\text { gorie Prozess, } \\
\text { Zähler }\end{array}$ & $\begin{array}{l}\text { Qualitäts- } \\
\text { messung, Ka- } \\
\text { tegorie Prozess, } \\
\text { Nenner }\end{array}$ \\
\hline $\begin{array}{l}\text { Nicht- } \\
\text { pharmakolo- } \\
\text { gische Be- } \\
\text { handlung }\end{array}$ & $\begin{array}{l}\text { Patient*innen } \\
\text { mit axSpA } \\
\text { werden über } \\
\text { den Nutzen } \\
\text { regelmäßiger } \\
\text { Bewegungs- } \\
\text { übungen } \\
\text { informiert }\end{array}$ & $\begin{array}{l}\text { Körperliche Aktivität sollte bei Patient*innen mit axSpA ein } \\
\text { integraler Bestandteil der Standardversorgung während des } \\
\text { gesamten Krankheitsverlaufs darstellen. Es ist wichtig, dass } \\
\text { Patient*innen mit axSpA über den Nutzen regelmäßiger Be- } \\
\text { wegungsübungen informiert werden, um Schmerzen und } \\
\text { Steifigkeit zu reduzieren. Außerdem können hierdurch die } \\
\text { kardiorespiratorische Fitness und Belastbarkeit verbessert } \\
\text { und damit auch das Risiko für Herz-Kreislauf-Erkrankungen } \\
\text { reduziert werden. Vorteile eines regelmäßigen Trainings soll- } \\
\text { ten dabei aktiv angesprochen werden, um die Patient*innen } \\
\text { zu unterstützen, ihre Funktionsfähigkeit zu verbessern und } \\
\text { ihre Lebensqualität zu erhalten }\end{array}$ & $\begin{array}{l}\text { Vorhaltung von } \\
\text { Strukturen und } \\
\text { Maßnahmen, um } \\
\text { Patient*innen mit } \\
\text { axSpA zu ermuti- } \\
\text { gen, regelmäßig } \\
\text { Bewegungsübun- } \\
\text { gen durchzufüh- } \\
\text { ren }\end{array}$ & $\begin{array}{l}\text { Die Anzahl der } \\
\text { Patient*innen } \\
\text { mit axSpA, die } \\
\text { über den Nutzen } \\
\text { regelmäßiger } \\
\text { Bewegungs- } \\
\text { übungen infor- } \\
\text { miert wurden }\end{array}$ & $\begin{array}{l}\text { Die Anzahl der } \\
\text { mit axSpA di- } \\
\text { agnostizierten } \\
\text { Patient*innen }\end{array}$ \\
\hline
\end{tabular}

Tab.8 Qualitätsstandard 7: Schulung als Basis zum Selbstmanagement

\begin{tabular}{|c|c|c|}
\hline Domäne & Standard & Rationale \\
\hline $\begin{array}{l}\text { Schulung als } \\
\text { Basis zum } \\
\text { Selbstma- } \\
\text { nagement }\end{array}$ & $\begin{array}{l}\text { Patient*innen } \\
\text { mit axSpA } \\
\text { wird innerhalb } \\
\text { von } 2 \text { Mona- } \\
\text { ten nach Dia- } \\
\text { gnosestellung } \\
\text { eine Schu- } \\
\text { lung über die } \\
\text { Erkrankung } \\
\text { angeboten } \\
\text { mit dem Ziel, } \\
\text { ein optimales } \\
\text { Selbstma- } \\
\text { nagement zu } \\
\text { erreichen }\end{array}$ & $\begin{array}{l}\text { Eine strukturierte Patient*innenschulung ist hilfreich, um } \\
\text { das Verständnis und das Selbstmanagement von axSpA zu } \\
\text { ermöglichen und das Risiko von Komplikationen zu reduzie- } \\
\text { ren. Es ist wichtig, dass die Patient*innen lernen, wie sie mit } \\
\text { ihren Symptomen umgehen, ihre Schmerzen und Sorgen } \\
\text { reduzieren und ihre Funktionsfähigkeit und Lebensqualität } \\
\text { verbessern können. Schulungsprogramme sollen Informa- } \\
\text { tionen über die Krankheit, den diagnostischen Abklärungs- } \\
\text { prozess, einen gesunden Lebensstil (körperliche Aktivität } \\
\text { und Raucherentwöhnung) und Behandlungsmöglichkeiten } \\
\text { einschließlich möglicher Nebenwirkungen enthalten. Medi- } \\
\text { zinisches Fachpersonal kann die Fähigkeit der Patient*innen, } \\
\text { ihren Zustand selbst zu steuern, unterstützen, indem es er- } \\
\text { mutigende Hinweise zur Ursache (Entzündung), aber auch } \\
\text { beruhigende Hinweise zum Verlauf der Erkrankung (Risiko } \\
\text { einer fortschreitenden Behinderung) und zur Bedeutung } \\
\text { eines aktiven Lebensstils gibt. Weitere Beratungen sollten } \\
\text { sich über den gesamten Krankheitsverlauf der Patient*innen } \\
\text { erstrecken }\end{array}$ \\
\hline
\end{tabular}

\begin{tabular}{|c|c|c|}
\hline $\begin{array}{l}\text { Qualitätsmes- } \\
\text { sung, Kategorie } \\
\text { Struktur }\end{array}$ & $\begin{array}{l}\text { Qualitätsmes- } \\
\text { sung, Kate- } \\
\text { gorie Prozess, } \\
\text { Zähler }\end{array}$ & $\begin{array}{l}\text { Qualitäts- } \\
\text { messung, Ka- } \\
\text { tegorie Prozess, } \\
\text { Nenner }\end{array}$ \\
\hline $\begin{array}{l}\text { Vorhaltung von } \\
\text { Strukturen und } \\
\text { Maßnahmen, um } \\
\text { sicherzustellen, } \\
\text { dass Angehörige } \\
\text { der Gesundheits- } \\
\text { berufe Zugang } \\
\text { zu Informationen } \\
\text { und Kenntnis- } \\
\text { sen haben, die } \\
\text { erforderlich sind, } \\
\text { um den Schu- } \\
\text { lungsbedarf der } \\
\text { Patient*innen } \\
\text { vollständig zu } \\
\text { decken und um } \\
\text { deren Fragen } \\
\text { beantworten zu } \\
\text { können }\end{array}$ & $\begin{array}{l}\text { Die Anzahl der } \\
\text { Patient*innen } \\
\text { mit einer dia- } \\
\text { gnostizierten } \\
\text { axSpA, die } \\
\text { innerhalb von } \\
2 \text { Monaten } \\
\text { nach Diagno- } \\
\text { sestellung eine } \\
\text { strukturierte Pa- } \\
\text { tient*innenschul } \\
\text { erhalten haben }\end{array}$ & $\begin{array}{l}\text { Die Anzahl der } \\
\text { mit axSpA di- } \\
\text { agnostizierten } \\
\text { Patient*innen }\end{array}$ \\
\hline
\end{tabular}

2 Mitglieder nicht an der Abstimmung teilnehmen.

\section{QS 6: Nicht-pharmakologische Behandlung}

Der Nutzen regelmäßiger Bewegung wurde während der Diskussion weiter spezifiziert, und schlussendlich einigte sich die Gruppe darauf, dass dem $^{\star}$ der Patient ${ }^{*}$ in Informationen über die regelmäßige Durchführung von Bewegungsübungen vermittelt werden sollten, da nicht jegliche Art der Bewegung, sondern v. a. gezielte Bewegungsübungen und regelmäßige körperliche Aktivität einen Benefit bei der Therapie der axSpA gezeigt haben ([23]; • Tab. 7).
Nach erfolgten Änderungen stimmten 15 von 15 Mitgliedern für die finale Übersetzung.

\section{QS 7: Patient*innenschulung}

Gemeinhin mag eine strukturierte $\mathrm{Pa}$ tient ${ }^{*}$ innenschulung sinnvoll erscheinen und ist auch von Expert*innen als solche angesehen, jedoch ist kritisch anzumerken, dass es bislang nur begrenzt wissenschaftliche Evidenz dafür gibt, eine strukturierte Schulung für das Krankheitsverständnis der Patient*innen als besser einzustufen als eine Informationsvermittlung durch den ${ }^{\star}$ die behandeln$\mathrm{de}^{*} \mathrm{n}$ Arzt $^{\star}$ Ärztin [24-27]. Strukturierte Schulungen sollen auch Hilfe zur Selbst- hilfe darstellen. Hierbei ist allerdings von erheblichen individuellen Unterschieden hinsichtlich des benötigten Schulungsbedarfes auszugehen. Insofern stellte sich die Frage, oblediglich die Anzahl strukturierter Patient*innenschulungen bereits ein Qualitätsmaß darstellt oder vielleicht doch eher das Ergebnis, wie z.B. das Wissen und das Krankheitsverständnis des $^{\star}$ der Patient ${ }^{\star}$ in. Da dies jedoch deutlich schwerer messbar ist und QS klar definierte messbare Prozess- und Strukturkategorien benötigen, wurde sich letztlich auf die Anzahl durchgeführter strukturierter Patient ${ }^{*}$ innenschulungen $2 \mathrm{Mo}$ nate nach Diagnosestellung als messbare Prozesskategorie geeinigt. 
Hier steht eine Anzeige.

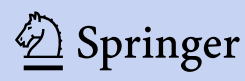


Qualitätsmessung, Kategorie Struktur

Vorhaltung von Strukturen und Maßnahmen, um sicherzustellen, dass Patient*innen mit axSpA innerhalb von 2 Werktagen nach Kontaktaufnahme von einem*einer Rheumatolog*in beraten werden
Qualitätsmes- Qualitätssung, Kategorie Prozess, Zähler messung, Ka-

Die Anzahl der Patient*innen mit axSpA, die einen Schub der Patient*innen, Erkrankung oder die um einen Nebenwirkun- dringenden Tergen der Medika- min gebeten tion haben und haben innerhalb von 2 Werktagen nach Kontaktaufnahme mit dem*der Rheumatolog*in beraten wurden

\section{Nenner}

Die Anzahl der mit axSpA diagnostizierte

.




\begin{tabular}{|c|c|}
\hline \multicolumn{2}{|c|}{ Abkürzungen } \\
\hline$A G$ & Arbeitsgruppe \\
\hline ASAS & $\begin{array}{l}\text { Assessment of SpondyloArthritis } \\
\text { International Society }\end{array}$ \\
\hline $\operatorname{axSpA}$ & Axiale SpA \\
\hline$D G R h$ & $\begin{array}{l}\text { Deutsche Gesellschaft für } \\
\text { Rheumatologie }\end{array}$ \\
\hline DMARD & $\begin{array}{l}\text { Disease-modifying anti-rheumatic } \\
\text { drug }\end{array}$ \\
\hline EULAR & $\begin{array}{l}\text { European League Against } \\
\text { Rheumatism }\end{array}$ \\
\hline$L L$ & Leitlinien \\
\hline NICE & $\begin{array}{l}\text { National Institute for Health and } \\
\text { Care }\end{array}$ \\
\hline NRS & Numerical rating skale \\
\hline$Q S$ & Qualitätsstandards \\
\hline$S p A$ & Spondyloarthritiden \\
\hline
\end{tabular}

Nach erfolgten Änderungen stimmten 15 von 15 Mitgliedern für die finale Übersetzung.

\section{Diskussion}

Dies ist die erste Definition der DGRh von Qualitätsstandards in der Rheumatologie. Auf Grundlage der vor Kurzem publizierten internationalen ASASEmpfehlungen für die Behandlung von axSpA-Patient ${ }^{*}$ innen [29] und der soeben aktualisierten nationalen Leitlinie für das Management von Patient ${ }^{*}$ innen mit axSpA [8] wurden 9 QS konsentiert, die messbar und geeignet sind, die Versorgungslage der Patient ${ }^{*}$ innen $\mathrm{zu}$ verbessern. Die Themen entsprechen denen in der Originalpublikation: Überweisung, Zeit bis zum Termin bei Rheumatolog*innen, diagnostische Abklärung durch Rheumatolog*innen, Monitoring der Erkrankung, leitliniengerechte Therapieeskalation, nichtpharmakologische Behandlung, $\mathrm{Pa}$ tient*innenschulung, Möglichkeit der kurzfristigen Terminvergabe und jährliche Beurteilung, hierbei geht es auch um Funktion, Mobilität und Komorbidität.

Bei den ersten 3 Punkten geht es v. a. um eine zeitnahe Diagnosestellung auf Expert ${ }^{*}$ innenbasis - die wesentliche Voraussetzung für eine adäquate und rechtzeitige Therapie. Hierbei spie- len die ASAS-Klassifikationskriterien von 2009 eine wichtige Rolle, die aber höchstens als grobe Orientierung dienen sollten [30]. Die Verfügbarkeit eines Magnetresonanztomographie(MRT)Geräts und radiologische Expertise sind zusätzliche Qualitätsmerkmale für diese Domänen. Die Definition eines Therapieziels, in der Regel Remission, ist essenziell für ein zielgerichtetes Monitoring, wobei es im Verlauf dann v. a. darum geht, das Erreichen dieses Ziels durch standardisierte Assessments zu überprüfen. Im Vordergrund steht dabei die Messung der Krankheitsaktivität durch BASDAI bzw. ASDAS-CRP [31]. Wird das Therapieziel nicht erreicht, folgt eine leitliniengerechte Therapieeskalation [8].

Die ersten Symptome bei Patient*innen mit axSpA beginnen durchschnittlich im 26. Lebensjahr $[1,2]$. Das bedeutet, dass es sich meist um Verläufe über mehrere Jahrzehnte handelt. Dabei ist auch von Bedeutung, dass die Morbidität und Mortalität für kardiovaskuläre Erkrankungen bei Patient*innen mit axSpA erhöht sind. Die Funktion der meisten Patient ${ }^{*}$ innen mit axSpA verschlechtert sich im Verlauf [32], wobei mit zunehmender Krankheitsdauer die strukturellen röntgenologisch erfassbaren Wirbelsäulenveränderungen eine größere Rolle spielen als die entzündlichen, die in früheren Phasen der Erkrankung eine größere Bedeutung haben [33]. Neben den medikamentösen Einflussmöglichkeiten spielt die Physiotherapie im Management eine große Rolle für die Erhaltung von Funktion und Beweglichkeit $[34,35]$. Dem wurde mit dem QS Nr. 6 Rechnung getragen. Hierzu, ebenso wie zum nächsten Thema, gehört auch die körperliche Aktivität, wozu es für $\mathrm{Pa}$ tient*innen mit muskuloskeletalen Erkrankungen aktuelle Empfehlungen der „European Alliance of Associations for Rheumatology“ (EULAR), früher „European League Against Rheumatism“ gibt [36].

Patient ${ }^{*}$ innenschulung ist ein zunehmend wichtiges Thema in der Rheumatologie, denn es ist davon auszugehen, dass ein $^{\star}$ e gut geschulte ${ }^{\star} r$ bzw. informierte ${ }^{\star} r$ Patient $^{*}$ in eine $^{*} n$ bessere ${ }^{*} n$ Partner ${ }^{*}$ in im Management auch im Sinne des „shared decision making“ darstellt. Die EULAR hat auch dazu Empfehlungen publiziert, und die DGRh hat sich in den letzten Jahren ebenfalls um eine Aktualisierung früherer Aktivitäten bemüht, erste Erfolge sind zu verzeichnen [37].

Auch bei Patient ${ }^{\star}$ innen mit axSpA gibt es Notfälle - wie etwa mögliche Medikamentennebenwirkungen, eine osteoporotische Wirbelkörperfraktur [38], eine akute Beteiligung des Atlantoaxialgelenks [39] oder neu aufgetretene kardiovaskuläre Komorbidität (z.B. arteriosklerotisch bedingte Ereignisse wie akuter Myokardinfarkt) [40-42]. Darüber hinaus gibt es aus Patient ${ }^{*}$ innensicht noch zahlreiche andere "Notfälle“, die den Wunsch nach einem schnellen Termin begründen können. Dieser Situation wurde mit dem QS 8 Rechnung getragen.

Der letzte QS 9 beinhaltet die jährliche Überprüfung von Funktion, Mobilität, Indikationsstellung für Röntgenuntersuchungen oder andere bildgebende Verfahren im Verlauf, eine Knochendichtemessung, des Impfstatus, kardiovaskuläre Komorbidität (z.B. Blutdruckmessung, Cholesterin usw., wenn von dem $^{\star}$ der Hausarzt*ärztin nicht bereits adressiert) und sozialmedizinische Aspekte (z. B. Antrag auf Rehabilitation, Antrag auf Schwerbehinderung, Rentenantrag usw.). Nicht nur hier kann man sich sicherlich Aufgaben für gut ausgebildete rheumatologische Fachassistent ${ }^{*}$ innen vorstellen.

Zusammengefasst gibt es jetzt erstmalig in Deutschland evidenzbasierte messbare Qualitätsstandards, die eine gute Zielformulierung und -struktur für zukünftige Verbesserungen der Versorgung bieten.

\section{Fazit für die Praxis}

\section{- In Deutschland bestehen nach wie vor Versorgungslücken in der Versorgung von axSpA-Patient*innen. \\ - Erstmalig wurden nun evidenzba- sierte Qualitätsstandards für die Be- handlung von axSpA-Patient*innen in Deutschland entwickelt.}




\section{- Die Versorgung von axSpA-Pa- tient*innen in Deutschland soll in Zukunft durch Verwendung von Qualitätsstandards messbar und das Outcome langfristig verbessert werden.}

\section{Korrespondenzadresse}

\section{PD Dr. med. U. Kiltz}

Rheumazentrum Ruhrgebiet, Herne, und RuhrUniversität Bochum

Claudiusstr. 45, 44649 Herne, Deutschland uta.kiltz@elisabethgruppe.de

Funding. Open Access funding enabled and organized by Projekt DEAL.

\section{Einhaltung ethischer Richtlinien}

Interessenkonflikt. U. Kiltz, V. Buschhorn-Milberger, K. Albrecht, H.-J. Lakomek, H.-M. Lorenz, M. Rudwaleit M. Schneider, H. Schulze-Koops, X. Baraliakos, F. Behrens, J. Brandt-Jürgens, H. Haibel, L. Hammel, K. Karberg, H. Kellner, D. Krause, U. Lange, E. Märker-Herrmann, D. Poddubnyy, J. Sieper, U. Syrbe und J. Braun geben an, dass kein Interessenkonflikt besteht.

Für diesen Beitrag wurden von den Autoren keine Studien an Menschen oder Tieren durchgeführt. Für die aufgeführten Studien gelten die jeweils dort angegebenen ethischen Richtlinien.

Open Access. Dieser Artikel wird unter der Creative Commons Namensnennung 4.0 International Lizenz veröffentlicht, welche die Nutzung, Vervielfältigung, Bearbeitung, Verbreitung und Wiedergabe in jeglichem Medium und Format erlaubt, sofern Sie den/die ursprünglichen Autor(en) und die Quelle ordnungsgemäß nennen, einen Link zur Creative Commons Lizenz beifügen und angeben, ob Änderungen vorgenommen wurden.

Die in diesem Artikel enthaltenen Bilder und sonstiges Drittmaterial unterliegen ebenfalls der genannten Creative Commons Lizenz, sofern sich aus der Abbildungslegende nichts anderes ergibt. Sofern das betreffende Material nicht unter der genannten Creative Commons Lizenz steht und die betreffende Handlung nicht nach gesetzlichen Vorschriften erlaubt ist, ist für die oben aufgeführten Weiterverwendungen des Materials die Einwilligung des jeweiligen Rechteinhabers einzuholen.

Weitere Details zur Lizenz entnehmen Sie bitte der Lizenzinformation auf http://creativecommons.org/ licenses/by/4.0/deed.de.

\section{Literatur}

1. Braun J, Sieper J (2007) Ankylosing spondylitis. Lancet 369(9570):1379-1390

2. Sieper J, Poddubnyy D (2017) Axial spondyloarthritis. Lancet 390(10089):73-84
3. Nikiphorou $E$, van der Heijde $D$, Norton $S$, Landewe RB, Molto A, Dougados M et al (2018) Inequity in biological DMARD prescription for spondyloarthritis across the globe: results from the ASAS-COMOSPA study. Ann Rheum Dis 77(3):405-411

4. Callhoff J, Albrecht K, Hoffmann F, Poddubnyy D, Gunther KP, Zink A (2019) Reality of care for musculoskeletal diseases at the population leve : results of the PROCLAIR collaborative project. ZRheumatol 78(Suppl 2):73-79

5. Regierer AC, Weiss A, Baraliakos X, Zink A, Listing J Strangfeld A (2020) Rabbit-SpA: a new disease register for axial spondyloarthritis and psoriatic arthritis. ZRheumatol 79(2):135-142

6. Redeker I, Callhoff J, Hoffmann F, Haibel H, Sieper J Zink A et al (2019) Determinants of diagnostic delay in axial spondyloarthritis: an analysis based on linked claims and patient-reported survey data. Rheumatology 58(9):1634-1638

7. Braun J, Schneider M, Lakomek HJ (2016) Cornerstones of quality assurance in medicine in Germany. Important impulse for the situation in treatment of rheumatism. Z Rheumatol 75(2):203-212

8. Kiltz U, Braun J, Becker A et al (2019) Long version on the S3 guidelines for axial spondyloarthritis including Bechterew's disease and early forms, Update 2019 : Evidence-based guidelines of the German Society for Rheumatology (DGRh) and participating medical scientific specialist societies and other organizations. Z Rheumatol 78(Suppl 1):3-64

9. Petzold T, Deckert S, Williamson PR, Schmitt J (2018) Quality measurement recommendations relevant to clinical guidelines in Germany and the United Kingdom: (what) can we learn from each other? Inquiry 55:46958018761495

10. Johansen I, Klokkerud M, Anke A, Borke JB, Glott T, Hauglie $U$ et al (2019) A quality indicator set for use in rehabilitation team care of people with rheumatic and musculoskeletal diseases; development and pilot testing. BMC Health Serv Res 19(1):265

11. Barber CEH, Mosher DP, Ahluwalia V, Zummer M, Marshall DA, Choquette D et al (2017) Development of a Canadian core clinical dataset to suppor high-quality care for Canadian patients with rheumatoid arthritis.J Rheumatol 44(12):1813-1822

12. Petersson IF, Strombeck B, Andersen L, Cimmino M, Greiff R, Loza E et al (2014) Development of healthcare quality indicators for rheumatoid arthritis in Europe: the eumusc.net project. Ann Rheum Dis 73(5):906-908

13. Navarro-Compan V, Smolen JS, Huizinga TW, Landewe R, Ferraccioli G, da Silva JA et al (2015) Quality indicators in rheumatoid arthritis: results from the Meteor database. Rheumatology 54(9):1630-1639

14. Kiltz U, Landewe RBM, van der Heijde D, Rudwaleit $\mathrm{M}$, Weisman $\mathrm{MH}$, Akkoc $\mathrm{N}$ et al (2020) Development of ASAS quality standards to improve the quality of health and care services for patients with axial spondyloarthritis. Ann Rheum Dis 79(2):193-201

15. Nice. Quality Standards process guide 2016.

16. Donabedian A (1980) Methods for deriving criteria for assessing the quality of medical care. Med Care Rev 37(7):653-698

17. Braun J, Robbers J, Lakomek HJ (2016) Quality of German medical services: a review. Z Rheumatol 75(1):97-102

18. Albrecht K, Huscher D (2017) Do we prescribe physical medicine sufficiently? Current data from the German Collaborative Arthritis Center. Akt Rheumatol 42:118-121

19. Jacobs H, Callhoff J, Albrecht K, Postler A, Saam J, Lange T et al (2020) Use of physical therapy in patients with osteoarthritis in Germany: an analysis of a linkage of claims and survey data. Arthritis Care Res 73(7):1013-1022

20. Zochling J, Bohl-Buhler MH, Baraliakos X, Feldtkeller E, Braun J (2006) Nonsteroidal antiinflammatory drug use in ankylosing spondylitis-a population-based survey. Clin Rheumatol 25(6):794-800

21. Redeker I, Callhoff J, Hoffmann F, Saam J, Haibel H, Sieper J et al (2020) Cost of illness in axial spondylarthritis for patients with and without tumor necrosis factor inhibitor treatment: results of a routine data analysis. ZRheumatol 79(1):85-94

22. Bundesamt S (2020) Angaben zur Krankenversicherung - (Ergebnisse des Mikrozensus) - Fachserie 13 Reihe 1.1 - 2019. https://www.destatis.de/ DE/Themen/Gesellschaft-Umwelt/Gesundheit/ Gesundheitszustand-Relevantes-Verhalten/ Publikationen/Downloads-Gesundheitszustand/ krankenversicherung-mikrozensus2130110199004.html. Zugegriffen: 27.12.2020

23. Klemm P, Hudowenz O, Asendorf T, Dischereit G, Muller-Ladner U, Lange U et al (2020) Evaluation of a special concept of physical therapy in spondyloarthritis: German multimodal rheumatologic complex treatment for spondyloarthritis. Clin Rheumatol 39(5):1513-1520

24. Bönisch A (2005) Evaluation eines Schulungsprogramms für Patienten mit Spondylitis ankylosans. Prädiktion, Verfahrensoptimierung und Kosten in der medizinischen Rehabilitation. Pertermann F, Regensburg, S51-102

25. Krauth C, Rieger J, Bönisch A, Ehlebracht-König I, Schwartz FW (2005) Gesundheitsökonomische Evaluation eines Patientenschulungsprogramms Spondylitis ankylosans in der stationären Rehabilitation. In: Petermann F (Hrsg) Prädiktion, Verfahrensoptimierung und Kosten in der medizinischen Rehabilitation. Roderer, Regensburg, S 103-143

26. Gerlich C, Andreica I, Kuffner R, Krause D, Lakomek HJ, Reusch A et al (2020) Evaluation of a basic educational program for patients with rheumatoid arthritis. ZRheumatol 79(8):737-748

27. Reusch A, Musekamp G, Kuffner R, Dorn M, Braun J, Ehlebracht-Konig I (2017) Evaluation of effectiveness of education in rheumatology : recommendations according to a patient education model.Z Rheumatol 76(7):613-621

28. Reusch A, Braun J, Lakomek HJ, Lakomek M, LindAlbrecht G, Janiche Hetal (2020) Flexible programs and advanced training for rheumatological patient education.Z Rheumatol 79(1):74-77

29. van derHeijdeD, Ramiro S, Landewe R, Baraliakos $X$ Van den Bosch F, Sepriano A et al (2017) 2016 update of the ASAS-EULAR management recommendations for axial spondyloarthritis. Ann Rheum Dis 76(6):978-991

30. Rudwaleit $M$, van der Heijde $D$, Landewe $R$, Listing J, Akkoc N, Brandt J et al (2009) The development of Assessment of SpondyloArthritis international Society classification criteria for axial spondyloarthritis (part II): validation and final selection. Ann Rheum Dis 68(6):777-783

31. Machado P, van der Heijde D (2011) How to measure disease activity in axial spondyloarthritis? Curr Opin Rheumatol 23(4):339-345

32. Landewe $R$, Dougados $M$, Mielants $H$, van der Tempel H, van der Heijde D (2009) Physical function in ankylosing spondylitis is indepen- 
dently determined by both disease activity and radiographic damage of the spine. Ann Rheum Dis 68(6):863-867

33. Machado P, Landewe R, Braun J, Hermann KG, Baker D, van der Heijde D (2010) Both structural damage and inflammation of the spine contribute to impairment of spinal mobility in patients with ankylosing spondylitis. Ann Rheum Dis 69(8):1465-1470

34. van Tubergen $A$, Landewe $R$, van der Heijde $D$, Hidding A, Wolter N, Asscher M et al (2001) Combined spa-exercise therapy is effective in patients with ankylosing spondylitis:arandomized controlled trial. Arthritis Rheum 45(5):430-438

35. Dagfinrud H, Kvien TK, Hagen KB (2008) Physiotherapy interventions for ankylosing spondylitis. Cochrane Database Syst Rev. https://doi.org/10. 1002/14651858.CD002822.pub3

36. Rausch Osthoff AK, Niedermann K, Braun J, Adams J, Brodin N, Dagfinrud H et al (2018) 2018 EULAR recommendations for physical activity in people with inflammatory arthritis and osteoarthritis. Ann Rheum Dis 77(9):1251-1260

37. Zangi HA, Ndosi M, Adams J, Andersen L, Bode C, Bostrom Cetal (2015) EULAR recommendationsfor patient education for people with inflammatory arthritis. Ann Rheum Dis 74(6):954-962

38. Prieto-Alhambra D, Munoz-Ortego J, De Vries $F$ Vosse D, Arden NK, Bowness P et al (2015) Ankylosing spondylitis confers substantially increased risk of clinical spine fractures: a nationwide casecontrol study. Osteoporos Int 26(1):85-91

39. Lee JS, Lee S, Bang SY, Choi KS, Joo KB, Kim YB et al (2012) Prevalence and risk factors of anterior atlantoaxial subluxation in ankylosing spondylitis. JRheumatol 39(12):2321-2326

40. Eriksson JK, Jacobsson L, Bengtsson K, Askling J (2017) Is ankylosing spondylitis a risk factor for cardiovascular disease, and how do these risks compare with those in rheumatoid arthritis? Ann Rheum Dis 76(2):364-370

41. Haroon NN, Paterson JM, Li P, Inman RD, Haroon N (2015) Patients with Ankylosing Spondylitis have increased cardiovascular and cerebrovascular mortality: a population-based study. Ann Intern Med 163(6):409-416

42. Molto A, Etcheto A, van der Heijde D, Landewe R, van den Bosch F, Bautista Molano W et al (2016) Prevalence of comorbidities and evaluation of their screening in spondyloarthritis: results of the international cross-sectional ASAS-COMOSPA study. Ann Rheum Dis 75(6):1016-1023
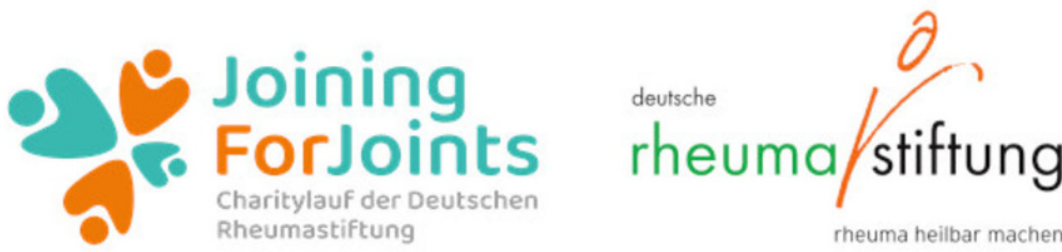

rheuma heilbar machen

\section{Erfolgreicher analoger Spendenlauf „JoiningForJoints" der Deutschen Rheumastiftung}

Rheuma heilbar machen ist das Ziel der Deutschen Rheumastiftung (DRS). Um auf die rheumatischen Erkrankungen aufmerksam zu machen und für Betroffene Spendengelder zu sammeln, fand am 2. September 2022 im Rahmen des 50. Deutschen Rheumatologiekongresses der analoge Spendenlauf JoiningforJoints statt. Rund 40 Teilnehmende absolvierten eine Laufstrecke von 5 Kilometern vom Estrel Hotel in der Sonnenallee aus entlang des Neuköllner Schifffahrtskanals zum Treptower Park inklusive der Umrundung des Karpfenteichs.

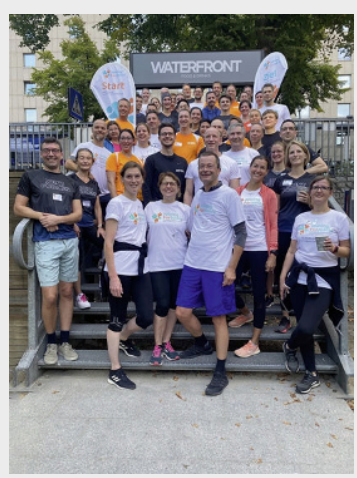

Großes Engagement beim analogen Spendenlauf am 12. September 2022 in Berlin: Dr. med. Florian Schuch (Mitte, 1. Reihe), Facharzt Rheumatologie und Vorstandsmitglied der Deutschen Rheumastiftung initiierte federführend den Spendenlauf JoiningForJoints. Zahlreiche Teilnehmende liefen für die, die aufgrund von Rheuma bewegungseingeschränkt sind.

Quelle: Deutsche Rheumastiftung

Der Startschuss zu diesem CharityLauf fiel bereits im April. Noch bis zum Weltrheumatag am 12. Oktober 2022 können Interessierte mitlaufen und/oder spenden. Zum Beispiel mit dem Kauf des Funktionslaufsshirts „JoingforJoints". „Dieser Lauf soll nun jedes Jahr stattfinden und so helfen, uns unserem Ziel, „Rheuma heilbar zu machen", Stück für Stück näher zu kommen.
Gleichzeitig wollen wir konsequent auf die Erkrankung und die damit verbundenen erheblichen Konsequenzen ins Bewusstsein der Öffentlichkeit tragen," so Dr. Florian Schuch, Rheumatologe und Vorstandsvorsitzender der Deutschen Rheumastiftung.

Unter dem Begriff Rheuma werden über 100 Krankheiten zusammengefasst. Allein ein Viertel aller Deutschen leidet beispielsweise an muskuloskelettalen Erkrankungen, die Bewegungen einschränken. „Jedes Jahr erkranken zwischen 60.000 bis 70.000 Menschen allein an rheumatoider Arthritis, einer Erkrankung, die Menschen vom Kindergarten bis ins hohe Alter treffen kann", erklärt Dr. Schuch. Mit den gesammelten Spenden wird die DRS Forschung, Aufklärung gefördert.

Weitere Informationen zum Spendenlauf unter

Charity Lauf der Deutschen Rheumastiftung JoiningForJoints www.joiningforjoints.org/ https://www.tshirt-drucker.de/c/charitylauf-dgrh

Deutsche Rheumastiftung

Dr. Florian Schuch, Deutsche Rheumastiftung Wilhelmine-Gemberg-Weg 6, Aufgang C

10179 Berlin

Tel: 030-24 048481

Fax: 030-24 048479

info@rheumastiftung.org

www.deutsche-rheumastiftung.de

Quelle: Deutsche Rheumastiftung 